\title{
Identification of candidate genes for milk production traits by RNA sequencing on bovine liver at different lactation stages
}

\author{
Qian $\mathrm{Li}^{1,2+}$, Ruobing Liang ${ }^{3 \dagger}$, Yan Liं, Yanxia Gao ${ }^{1}$, Qiufeng $\mathrm{Li}^{1}$, Dongxiao $\mathrm{Sun}^{3 *}$ (D) and Jianguo $\mathrm{Li}^{1 *}$
}

\begin{abstract}
Background: RNA-sequencing was performed to explore the bovine liver transcriptomes of Holstein cows to detect potential functional genes related to lactation and milk composition traits in dairy cattle. The bovine transcriptomes of the nine liver samples from three Holstein cows during dry period (50-d prepartum), early lactation (10-d postpartum), and peak of lactation (60-d postpartum) were sequenced using the Illumina HiSeq 2500 platform.

Results: A total of 204, 147 and 81 differentially expressed genes (DEGs, $p<0.05$, false discovery rate $q<0.05$ ) were detected in early lactation vs. dry period, peak of lactation vs. dry period, and peak of lactation vs. early lactation comparison groups, respectively. Gene ontology and KEGG pathway analysis showed that these DEGs were significantly enriched in specific biological processes related to metabolic and biosynthetic and signaling pathways of PPAR, AMPK and p53 ( $p<0.05)$. Ten genes were identified as promising candidates affecting milk yield, milk protein and fat traits in dairy cattle by using an integrated analysis of differential gene expression, previously reported quantitative trait loci (QTL), data from genome-wide association studies (GWAS), and biological function information. These genes were APOC2, PPP1R3B, PKLR, ODC1, DUSP1, LMNA, GALE, ANGPTL4, LPIN1 and CDKN1A.
\end{abstract}

Conclusion: This study explored the complexity of the liver transcriptome across three lactation periods in dairy cattle by performing RNA sequencing. Integrated analysis of DEGs and reported QTL and GWAS data allowed us to find ten key candidate genes influencing milk production traits.

Keywords: RNA-sequencing, Liver, Candidate gene, Milk production traits, Dairy cattle

\footnotetext{
* Correspondence: sundx@cau.edu.cn; jgli@hebau.edu.cn

${ }^{+}$Qian Li and Ruobing Liang contributed equally to this work.

${ }^{3}$ Department of Animal Genetics, Breeding and Reproduction, College of Animal Science and Technology, Key Laboratory of Animal Genetics and Breeding of Ministry of Agriculture, National Engineering Laboratory of Animal Breeding, China Agricultural University, No.2 Yuanmingyuan West Road, Beijing 100193, China

${ }^{1}$ College of Animal Science and Technology, Hebei Agricultural University, Lekai South Street, Baoding 071001, China

Full list of author information is available at the end of the article
}

(c) The Author(s). 2020 Open Access This article is licensed under a Creative Commons Attribution 4.0 International License, which permits use, sharing, adaptation, distribution and reproduction in any medium or format, as long as you give appropriate credit to the original author(s) and the source, provide a link to the Creative Commons licence, and indicate if changes were made. The images or other third party material in this article are included in the article's Creative Commons licence, unless indicated otherwise in a credit line to the material. If material is not included in the article's Creative Commons licence and your intended use is not permitted by statutory regulation or exceeds the permitted use, you will need to obtain permission directly from the copyright holder. To view a copy of this licence, visit http://creativecommons.org/licenses/by/4.0/. The Creative Commons Public Domain Dedication waiver (http://creativecommons.org/publicdomain/zero/1.0/) applies to the data made available in this article, unless otherwise stated in a credit line to the data. 


\section{Background}

In dairy cattle, milk yield and compositions are the most important economical traits that are typical quantitative characteristics controlled by multiple QTLs and polygenes simultaneously. Detection of key functional genes or causal variations on milk production traits could provide valuable molecular information for marker-assisted selection or genomic selection in dairy cattle thereby greatly shortening generation interval and increase the rate of genetic gain through pre-selecting young candidate bulls prior to progeny testing. In the past several decades, extensive quantitative trait locus (QTL) mapping, candidate genes analysis, and genome-wide association study (GWAS) have been implemented to identify QTLs, genes and mutations with large effects on the milk traits of dairy cows [1-4]. As of December 29, 2019, a large number of QTLs and genetic associations have been detected with 5093, 19,782, and 22,418 loci for milk yield, milk protein and milk fat, respectively (http://www.animalgenome.org/cgi-bin/QTLdb/). However, only a few studies, including DGAT1 (Diacylglycerol O-Acyltransferase 1), GHR (Growth Hormone Receptor), and ABCG2 (ATP Binding Cassette Subfamily $G$ Member 2) gene have been confirmed as major genes for milk production traits in dairy cattle $[2,5,6]$.

Beyond marker-QTL linkage analysis (LA) and/or linkage disequilibrium (LD) and GWAS, with the dramatically cost reductions and rapid development of next generation sequencing, RNA-sequencing (RNA-Seq) has become a commonly used approach to identify candidate genes for complex traits in human and domestic animals in recent years. Many bovine studies utilizing RNA-Seq have been conducted [7], including mammary tissue [8], embryos [9], leukocytes [10], different disease conditions [11], or factors with various nutrition traits [12]. Our previous RNA-Seq studies with mammary gland tissues from lactating Holstein cows with extremely high and low milk protein and fat percentages and from cows in non-lactation and peak of lactation identified 17 differentially expressed genes (DEGs) as promising candidates for milk composition traits $[8,13]$. However, only a limited number of studies on transcriptional profiles in bovine liver have been reported until now $[12,14]$.

Actually, besides mammary gland epithelium, liver also plays critical roles in milk synthesis during lactation as the most important organ where the metabolism of gluconeogenesis, lipid, amino acids and other substances takes place in dairy cows [15-17]. Gluconeogenesis in liver is vital to meet glucose requirements for dairy cows in perinatal period. Dorland et al. (2009) presented the liver, as a crucial role in considerable metabolic adaptation, supported pregnancy and lactation through coordination and interconversion of nutrients [17], especially during the period of transition from late gestation to early lactation. A few genes in bovine liver involved in glucose and lipid metabolism had been detected via bovine gene microarrays [18]. Smith et al. (1998) reported that cholesterol content was increased in liver to meet lipoproteins synthesis and secretion, and then provided the mammary gland with cholesterol and triglycerides after parturition [19]. The mRNA expression of pyruvate carboxylase (PC) increased in medium and high liver fat concentration (LFC) groups than that in the low-LFC group, suggesting expressions of more genes related to gluconeogenesis than genes for lipid metabolism in early lactation [20]. Bu et al. (2017) compared the gene expression profiles between liver and mammary tissues during lactation in cows and found that liver expressed a larger number of metabolic genes, especially related to lipid, while mammary gland had more genes with regards to protein synthesis and secretion, proliferation/ differentiation [21]. Our previous study detected some differentially expressed mRNAs, miRNAs and lncRNAs among different lactation stages in liver thereby predicted the competing endogenous RNAs (ceRNA) regulatory networks in Holstein cow [22].

In view of the important roles of liver in metabolism, the objective of this study was to search for key functional genes for milk production traits by performing RNA sequencing of liver samples from dairy cows in non-lactation and lactation. Here, we utilized liver biopsy transcriptome data from three Holstein cows during the dry period, early lactation and peak of lactation obtained using RNA-Seq and identified ten promising candidate genes for milk yield and milk compositions (protein and fat) in Holstein. Our findings could provide new insights into elucidation of the genetic basis for milk traits and potential molecular information for genomic selection in dairy cattle breeding.

\section{Methods}

\section{Animals and liver tissue sample collection}

Three healthy Chinese Holstein cows with similar body weight, milk yield, and milk composition were selected from 1300 Chinese Holstein cows fed in Baoding Hongda Animal Husbandry Limited Company in Hebei Province (Baoding, China) [22]. Liver biopsies were collected on 50-d before parturition (dry period), 10-d after parturition (early lactation) and 60-d after parturition (peak of lactation) from each cow as described in detail in our previous study [22]. As a result, a total of nine samples were obtained. All protocols for collection of the tissues of experimental individuals were reviewed and approved by Animal Welfare Committee of Hebei Agricultural University. All animals care and treatment in compliance with the "Principles of Laboratory Animal Care (NIH Publication No. 86-23, revised 1985)". The 
cows after study were all alive and healthy and still produced milk.

\section{RNA sequencing, read alignment and identification of differentially expressed genes}

The total RNA was isolated from each live tissue sample with TRIzol reagent (Life Technologies, CA, USA) and purified with RNase-free DNase (TIANGEN, Beijing, China). RNA integrity number (RIN) was detected with RNA Nano 6000 Assay Kit on Bioanalyzer 2100 system (Agilent Technologies, CA, USA) and samples with RIN values of higher than 7.0 were used as input material for the RNA sample preparations. Subsequently, sequencing libraries were generated using NEBNext ${ }^{\circ}$ Ultra $^{\mathrm{Tm}}$ Directional RNA Library Prep Kit for Illumina (New England Biolabs, MA, USA) and sequenced on Illumina HiSeq 2500 platform. After removing adapters, deleting reads containing poly- $\mathrm{N}$ and low quality reads from the raw data, we aligned the paired-end reads of the clean data to the cattle reference genome assembly UMD 3.1.80. Detailed descriptions for sequencing and assembling were shown previously [22].

Gene expression level was calculated as fragments per kilo base pair $(\mathrm{kb})$ of transcript per million mapped fragments (FPKM). DEGs among the different lactation stages were detected and quantified using Cuffdiff (http://cole-trapnell-lab.github.io/cufflinks/cuffdiff/).

FPKM value, fold changes (in log2 scale), $p$-values and q-values (false discovery rate: corrected p-values) of DEGs were reported in the output files from Cuffdiff, and q-value of $<0.05$ was set as the threshold for significantly differential gene expression.

\section{Functional enrichment analysis of differentially expressed genes}

Ensembl Gene IDs were uploaded to the DAVID Functional Annotation Tool (https://david.ncifcrf.gov/home. jsp) and Gene Ontology (GO) functional annotation and Kyoto Encyclopedia of Genes and Genomes (KEGG) were performed. $P$-value of 0.05 was set as the threshold for significantly enriched GO terms and pathways.

\section{Integrated analysis of the differentially expressed genes and previously reported QTL and GWAS data}

To better identify candidate genes for milk traits, we further compared the physical positions on genome of DEGs with the previously reported QTLs that have been shown to be associated with five milk production traits, namely milk yield, protein yield, fat yield, protein percentage and fat percentage (Cattle QTLdb: http://www. animalgenome.org/cgi-bin/QTLdb/BT/index) and the significant SNPs for five milk traits identified by previous GWAS in dairy cattle [23]. Thereby, DEGs close to the
QTL peak positions (less than $5 \mathrm{cM}$ ) and near significant SNPs (less than $5 \mathrm{Mb}$ ) were selected as candidate genes.

\section{Results \\ Overview of RNA sequencing}

We sequenced the cDNA libraries of nine liver tissue samples from three Holstein cows with three samples from each period, i.e. dry period (50-d prepartum), early lactation (10-d postpartum) and peak of lactation (60-d postpartum) using HiSeq 2500. In total, we acquired 780.38 million clean reads, with 86.71 million for each sample on average (range from 78.62 to 97.78 million). The sequencing quality values of Q20 and Q30 were 93.13 and $87.48 \%$ respectively (Additional file 1 ) indicating a high quality of sequencing data. Alignment of the sequencing reads against the bovine genome UMD3.1.80 yielded $84.49 \%$ of uniquely aligned reads across the nine samples (Additional file 2) which were used for further analyses. Pearson correlation between the three samples in each stage was higher than 0.935 that was considered as a high correlation indicating the high similarity of biological replicates (Additional file 3).

\section{Differentially expressed genes across three periods}

With Cuffdiff software, a total of 204 (118 up-regulated and 86 down-regulated), 147 (106 up-regulated and 41 down-regulated) and 81 (57 up-regulated and 24 downregulated) differentially expressed genes (DEGs, $p<0.05$, false discovery rate $\mathrm{q}<0.05$ ), which were ranked in the top half expressed genes, were detected in the early lactation vs. dry period, peak of lactation vs. dry period, and peak of lactation vs. early lactation, respectively (Additional file 4 and Additional file 5). Details of the top 10 DEGs in the three comparisons and their full name as well as q-value and fold change were described in Table 1.

\section{Gene ontology enrichment and pathway analysis}

To further know about the functional associations of the differentially expressed genes between lactation periods, we implemented gene ontology (GO) analysis with DAVID software (Additional file 6). Between early lactation and dry period, significantly enriched GO terms $(p<0.05)$ were mainly focused on metabolism-related functions, especially on carboxylic acid metabolic process, transport of lipid/ cholesterol/ sterol, regulation of cellular ketone metabolic process/ lipid biosynthetic process/ lipoprotein lipase activity, high-density lipoprotein (HDL) particle, lipoprotein particle, very low-density lipoprotein (VLDL) particle and small molecule/ carboxylic acid/ carbohydrate catabolic process. Of these, insulin-like growth factor binding and anion binding were the most significantly enriched GO molecular functions. Of note, the top 10 DEGs were involved in 
Table 1 Top 10 differentially expressed genes between each comparison of RNA-sea

\begin{tabular}{|c|c|c|c|}
\hline Gene name & Gene description (early lactation vs. dry period) & FDR (q-value) & Log $_{2}$ FoldChange \\
\hline LRRC73 & leucine rich repeat containing 73 & 0.004690 & inf \\
\hline GPX3 & glutathione peroxidase 3 & 0.004690 & 5.57 \\
\hline APOA4 & apolipoprotein A4 & 0.004690 & 4.26 \\
\hline HP & haptoglobin & 0.004690 & 4.07 \\
\hline MFSD2 & major facilitator superfamily domain containing $2 \mathrm{~A}$ & 0.004690 & 3.67 \\
\hline CDC42EP5 & CDC42 effector protein 5 & 0.004690 & 3.46 \\
\hline SLC13A5 & solute carrier family 13 & 0.011199 & 3.22 \\
\hline SMCT1 & solute carrier family 5 & 0.004690 & 3.22 \\
\hline PAQR9 & progestin and adipoQ receptor family member IX & 0.004690 & 3.14 \\
\hline SFRP2 & secreted frizzled-related protein 2 & 0.008110 & -3.62 \\
\hline Gene name & Gene description (peak of lactation vs. dry period) & $\begin{array}{l}\text { FDR } \\
\text { (q-value) }\end{array}$ & Log $_{2}$ FoldChange \\
\hline ISG15 & ISG15 ubiquitin like modifier & 0.004690 & 5.09 \\
\hline IFIT1 & interferon-induced protein with tetratricopeptide repeats 1 & 0.004690 & 4.98 \\
\hline RSAD2 & radical S-adenosyl methionine domain containing 2 & 0.004690 & 4.24 \\
\hline APOA4 & apolipoprotein A4 & 0.004690 & 3.64 \\
\hline MX1 & MX dynamin like GTPase 1 & 0.004690 & 3.61 \\
\hline GPX3 & glutathione peroxidase 3 & 0.004690 & 3.54 \\
\hline MX2 & MX dynamin like GTPase 2 & 0.004690 & 3.42 \\
\hline USP18 & ubiquitin specific peptidase 18 & 0.004690 & 3.39 \\
\hline LOC100298356 & bone marrow stromal antigen 2 & 0.004690 & 3.33 \\
\hline HERC6 & HECT and RLD domain containing E3 ubiquitin protein ligase family member 6 & 0.004690 & 3.15 \\
\hline Gene name & Gene description (peak of lactation vs. early lactation) & FDR (q-value) & $\log _{2}$ FoldChange \\
\hline ISG12(B) & TLH29 protein precursor-like & 0.004690 & 4.26 \\
\hline RSAD2 & radical S-adenosyl methionine domain containing 2 & 0.004690 & 4.13 \\
\hline IFIT1 & interferon-induced protein with tetratricopeptide repeats 1 & 0.004690 & 4.12 \\
\hline ISG15 & ISG15 ubiquitin like modifier & 0.004690 & 3.17 \\
\hline FKBP5 & FKBP prolyl isomerase 5 & 0.004690 & 2.48 \\
\hline MX1 & MX dynamin-like GTPase 1 & 0.017022 & 2.42 \\
\hline RXRG & retinoid $\mathrm{X}$ receptor, gamma & 0.004690 & -2.42 \\
\hline ITGAD & intrgrin, alpha D & 0.004690 & -2.48 \\
\hline LYZ2 & lysozyme C-2 & 0.011199 & -2.81 \\
\hline$H B B$ & hemoglobin, beta & 0.004690 & -5.54 \\
\hline
\end{tabular}

response to metabolic process and transport, cellular component organization localization.

Comparing the peak of lactation with dry period, the most significantly enriched GO categories $(p<0.05)$ were related to response to biotic stimulus/ other organism/ external biotic stimulus, immune effector process, HDL particle, plasma lipoprotein particle, lipoprotein particle, and molecular functions associated with doublestranded RNA binding, insulin-like growth factor binding, and growth factor binding. The top 10 DEGs were related to response to stimulus, metabolic process, biological regulation, cellular component organization or biogenesis, multi-organism process, and localization.

However, between peak of lactation and early lactation, the main significant GO categories $(p<0.05)$ were focused on triglyceride/ acylglycerol/ neutral lipid/ fatty acid/ monocarboxylic acid metabolic process, and molecular functions associated with fructose transmembrane transporter activity and double-stranded RNA binding. Among the TOP10 DEGs, cellular and metabolic processes, response to stimulus, biological regulation, cellular component organization or biogenesis, multi-organism process, and localization were enriched.

In addition, KEGG analysis significantly enriched 53, 39 and 46 pathways (Additional file 7), including PPAR and AMPK signaling pathways, cholesterol/ fatty acid metabolism and glycolysis/gluconeogenesis between early lactation vs. dry period, and PPAR, p53 and AMPK signaling pathways between peak of lactation and other two periods. 
Candidate genes identified by integrated analysis of RNASeq, reported QTL and GWAS data

To identify candidate functional genes affecting milk production traits, integrated analysis of the RNA-Seq data in this study and the previously reported genetic data including QTL mapping and GWASs was performed. First, we compared the physical position of each DEG with the position of known QTLs that have been shown to be associated with milk yield, milk protein and milk fat traits in dairy cattle from the Cattle QTLdb database (http://www.animalgenome.org/cgi-bin/QTLdb/). Then, each DEG was compared with the significant SNPs for milk traits in Holstein identified in a GWA study by Cole et al. [23]. As a result, ten common differentially expressed genes that were simultaneously close to the known QTLs (less than $5 \mathrm{cM}$ ) and significant SNPs (less than $5 \mathrm{Mb}$ ) were identified (Tables 2 and 3).

Thus, through combination of DEGs, QTL and GWAS data, and biological functions, such ten genes were suggested as promising candidates for milk production traits, i.e. APOC2 (Apolipoprotein C2), PPP1R3B (Protein phosphatase 1 regulatory subunit $3 B)$, PKLR (Pyruvate kinase), ODC1 (Ornithine decarboxylase 1), DUSP1 (Dual specificity phosphatase 1) and LMNA (Lamin), GALE (UDP-galactose-4-epimerase), ANGPTL4 (Angiopoietin like 4),
LPIN1 (Lipin 1), and CDKN1A (Cyclin dependent kinase inhibitor 1A).

\section{Discussion}

In this study, we obtained the liver transcriptome profiles in different lactation periods of Holstein cows using high-throughput RNA sequencing, and 432 differentially expressed genes (DEGs), which were ranked in the top half expressed genes, were detected among the dry period ( -50 day), early lactation (+ 10 day), and peak of lactation (+ 60 days). The DEGs expressed in the bottom half level were eliminated to ensure the detection power. Rapaport et al. (2013) investigated the relationship between detection power of DEGs and sequence depth and number of replicates, and demonstrated that with most methods, over $90 \%$ of differently expressed genes at the top expression levels are detected with little as 2 replicates and 5\% of the reads [24]. Trapnell et al. (2013) also reported that the detection rate of DEGs was similar for three or more replicates using Cuffdiff [25]. Of noted, the more biological replicates are taken, more detection power are improved.

Through functional enrichment, we found that the differentially expressed genes (DEGs) between milking and non-lactation status (early and peak of lactation vs

Table 2 Detailed information on the reported QTLs containing the 11 DEGs in bovine liver at three lactation stages

\begin{tabular}{|c|c|c|c|c|c|c|c|c|c|}
\hline \multirow{2}{*}{$\begin{array}{l}\text { Gene } \\
\text { name }\end{array}$} & \multirow{2}{*}{$\begin{array}{l}\text { Early } \\
\text { vs. } \\
\text { dry }\end{array}$} & \multirow{2}{*}{$\begin{array}{l}\text { Peak } \\
\text { vs. } \\
\text { dry }\end{array}$} & \multirow{2}{*}{$\begin{array}{l}\text { Peak } \\
\text { vs. } \\
\text { early }\end{array}$} & \multirow[t]{2}{*}{ Position (bp) } & \multirow{2}{*}{$\begin{array}{l}\text { Position } \\
(\mathrm{cM})^{\mathrm{a}}\end{array}$} & \multicolumn{4}{|l|}{ Previously reported QTL } \\
\hline & & & & & & Distance to QTL peak (cM) & $\mathrm{Cl}$ and peak location (CM) & Trait & QTL ID \\
\hline \multirow[t]{2}{*}{ APOC2 } & + & & & $53,057,717-53,059,957$ & Chr18:67.9 & 1.9 & 54.713-76.57 (peak:66) & PY & 2721 \\
\hline & & & & & & 2.3 & 54.713-76.57 (peak:65.6) & MY & 1532 \\
\hline ACADVL & + & & & $27,568,181-27,573,378$ & Chr19:47.1 & 4.0 & 14.1-60.4 (peak:43.1) & FY & 10,443 \\
\hline PPP1R3B & - & & & $24,316,623-24,317,477$ & Chr27:34.3 & 0.5 & 15.176-52.32 (peak:33.8) & FP & 2740 \\
\hline GALE & + & & & $129,706,509-129,711,871$ & Chr2:121.9 & 1.9 & 115.437-130 (peak:120) & FY & 1515 \\
\hline \multirow[t]{3}{*}{$P K L R$} & - & & & $15,396,974-15,408,994$ & Chr3:25.7 & 0.7 & 22.61-27.41 (peak:25) & FY & 2655 \\
\hline & & & & & & 0.7 & 22.61-27.41 (peak:25) & PP & 2656 \\
\hline & & & & & & 1.7 & 6-32 (peak:27.41) & PP & 3435 \\
\hline \multirow[t]{2}{*}{ ANGPTL4 } & + & + & & $18,236,482-18,243,588$ & Chr7:20.6 & 2.8 & 16.75-39.33 (peak:17.8) & PP & 3534 \\
\hline & & & & & & 4.7 & 16.75-39.33 (peak:15.9) & PP & 3536 \\
\hline \multirow[t]{2}{*}{ CDKNIA } & - & & + & $10,551,755-10,568,782$ & Chr23:14.1 & 0.9 & 11.82-20.66 (peak:15) & MY & 2590 \\
\hline & & & & & & 1.0 & 13.77-28.30 (peak:15.1) & MY & 2568 \\
\hline ODC1 & + & + & & $87,175,389-87,182,661$ & Chr11:95.0 & 0.1 & 92.17-97.57 (peak:94.9) & FY & 2669 \\
\hline LPIN1 & & + & + & $86,050,742-86,128,538$ & Chr11:93.8 & 1.1 & 92.17-97.57 (peak:94.9) & FY & 2669 \\
\hline \multirow[t]{2}{*}{ DUSP1 } & & + & & $4,449,106-4,452,189$ & Chr20:5.5 & 2.7 & 0-31.86 (peak:8.238) & FY & 2564 \\
\hline & & & & & & 4.6 & 0-20.165 (peak:10.08) & PY & 2750 \\
\hline \multirow[t]{3}{*}{ LMNA } & + & & & $14,695,234-14,724,663$ & Chr3:24.1 & 0.5 & 22.61-27.41 (peak:25) & FY & 2655 \\
\hline & & & & & & 0.5 & 22.61-27.41 (peak:25) & PP & 2656 \\
\hline & & & & & & 2.9 & 6-32 (peak:27.41) & PP & 3435 \\
\hline
\end{tabular}

${ }^{a}$ The linkage position was estimated relative to UMD3.1.80 and based on the QTL mapper v.2.019 at www.animalgenome.org/cgi-bin/QTLdb/. MY: milk yield; PY: milk protein yield; FY: milk fat yield; PP: milk protein percentage; FP: milk fat percentage 
Table 3 Detailed information on the nearest and most significant SNPs from previous GWAS to the 22 differentially expressed genes in bovine liver at different lactation stages

\begin{tabular}{|c|c|c|c|c|c|c|c|c|c|}
\hline \multirow{2}{*}{$\begin{array}{l}\text { Gene } \\
\text { name }\end{array}$} & \multirow{2}{*}{$\begin{array}{l}\text { Early } \\
\text { vs. } \\
\text { dry }\end{array}$} & \multirow{2}{*}{$\begin{array}{l}\text { Peak } \\
\text { vs. } \\
\text { dry }\end{array}$} & \multirow{2}{*}{$\begin{array}{l}\text { Peak } \\
\text { vs. } \\
\text { early }\end{array}$} & \multirow[t]{2}{*}{ Gene position $(b p)^{a}$} & \multicolumn{3}{|c|}{ Nearest and most significant SNPs of GWAS } & \multirow[t]{2}{*}{ Traits } & \multirow[t]{2}{*}{ Raw $p$ value } \\
\hline & & & & & Distance & Name & $\begin{array}{l}\text { Position } \\
(\mathrm{bp})^{\mathrm{b}}\end{array}$ & & \\
\hline \multirow[t]{2}{*}{ APOA1 } & + & & - & $\begin{array}{l}\text { Chr15:27932198-27, } \\
934,085\end{array}$ & $1.77 \mathrm{Mb}$ & BTB-00590603 & $29,702,877$ & FY,PY,FP,PP & $\begin{array}{l}7.05 \mathrm{E}-22 \sim \\
4.71 \mathrm{E}-16\end{array}$ \\
\hline & & & & & $3.59 \mathrm{Mb}$ & BTB-00590405 & $31,527,773$ & FY,PY & $\begin{array}{l}4.12 \mathrm{E}-11 \sim \\
1.22 \mathrm{E}-10\end{array}$ \\
\hline \multirow[t]{7}{*}{ ABCG8 } & - & & & $\begin{array}{l}\text { Chr11: 26156365-26, } \\
175,034\end{array}$ & $1.34 \mathrm{Mb}$ & BTB-00470332 & $27,519,615$ & FY,PY & $\begin{array}{l}3.66 \mathrm{E}-09 \sim \\
1.25 \mathrm{E}-08\end{array}$ \\
\hline & & & & & $1.78 \mathrm{Mb}$ & BFGL-NGS-115431 & $24,379,969$ & $\begin{array}{l}\text { MY,FY,PY, } \\
F P, P P\end{array}$ & $\begin{array}{l}3.18 \mathrm{E}-29 \sim \\
1.92 \mathrm{E}-07\end{array}$ \\
\hline & & & & & $2.46 \mathrm{Mb}$ & BTB-01556917 & $23,700,685$ & PP & $3.46 \mathrm{E}-12$ \\
\hline & & & & & $2.56 \mathrm{Mb}$ & Hapmap59290-rs29022016 & $23,599,074$ & PY,PP & $\begin{array}{l}1.87 \mathrm{E}-22 \sim \\
7.11 \mathrm{E}-08\end{array}$ \\
\hline & & & & & $3.49 \mathrm{Mb}$ & BFGL-NGS-116483 & $22,666,445$ & $\begin{array}{l}\text { MY,FY,PY, } \\
\text { FP,PP }\end{array}$ & $\begin{array}{l}1.60 \mathrm{E}-24 \sim \\
1.14 \mathrm{E}-08\end{array}$ \\
\hline & & & & & $3.57 \mathrm{Mb}$ & ARS-BFGL-NGS-43804 & $22,588,525$ & MY,FY,PY,FP & $\begin{array}{l}2.85 \mathrm{E}-26 \sim \\
5.15 \mathrm{E}-08\end{array}$ \\
\hline & & & & & $4.03 \mathrm{Mb}$ & ARS-BFGL-NGS-100459 & $22,128,569$ & FY,PY,PP & $\begin{array}{l}1.03 \mathrm{E}-24 \sim \\
7.98 \mathrm{E}-08\end{array}$ \\
\hline \multirow[t]{4}{*}{ APOC2 } & + & & & $\begin{array}{l}\text { Chr18: } 53057717-53 \text {, } \\
059,957\end{array}$ & $\begin{array}{l}888.61 \\
\mathrm{~Kb}\end{array}$ & BFGL-NGS-117985 & $53,948,569$ & $\begin{array}{l}\text { MY,FY,PY, } \\
\text { FP,PP }\end{array}$ & $\begin{array}{l}9.83 \mathrm{E}-55 \sim \\
1.71 \mathrm{E}-19\end{array}$ \\
\hline & & & & & $910.9 \mathrm{~Kb}$ & Hapmap41540-BTA-43915 & $53,970,861$ & $F Y, P Y, F P$ & $\begin{array}{l}3.07 \mathrm{E}-18 \sim \\
2.19 \mathrm{E}-09\end{array}$ \\
\hline & & & & & $2.83 \mathrm{Mb}$ & Hapmap47618-BTA-43817 & $55,892,476$ & $\mathrm{PP}$ & 1.07E-08 \\
\hline & & & & & $4.11 \mathrm{Mb}$ & ARS-BFGL-NGS-98028 & $57,174,711$ & FY,PY,FP,PP & $\begin{array}{l}1.40 \mathrm{E}-21 \sim \\
1.04 \mathrm{E}-10\end{array}$ \\
\hline \multirow[t]{2}{*}{ APOA4 } & + & + & & $\begin{array}{l}\text { Chr15: 27906598-27, } \\
919,592\end{array}$ & $1.78 \mathrm{Mb}$ & BTB-00590603 & $29,702,877$ & FY,PY,FP,PP & $\begin{array}{l}7.05 E-22 \sim \\
4.71 E-16\end{array}$ \\
\hline & & & & & $3.61 \mathrm{Mb}$ & BTB-00590405 & $31,527,773$ & FY,PY & $\begin{array}{l}4.12 \mathrm{E}-11 \sim \\
1.22 \mathrm{E}-10\end{array}$ \\
\hline \multirow[t]{2}{*}{ SAA1 } & + & + & & $\begin{array}{l}\text { Chr29:26696726-26, } \\
714,918\end{array}$ & $6.41 \mathrm{~Kb}$ & ARS-BFGL-NGS-24998 & $26,721,324$ & $\mathrm{PP}$ & $2.96 \mathrm{E}-08$ \\
\hline & & & & & $4.19 \mathrm{Mb}$ & UA-IFASA-8605 & $30,901,735$ & $F Y, F P, P P$ & $\begin{array}{l}1.50 \mathrm{E}-16 \sim \\
1.50 \mathrm{E}-07\end{array}$ \\
\hline \multirow[t]{3}{*}{$P C$} & + & & & $\begin{array}{l}\text { Chr29: 45508279-45, } \\
611,042\end{array}$ & $2.89 \mathrm{Mb}$ & ARS-BFGL-NGS-2893 & $48,501,273$ & FY,PY,FP,PP & $\begin{array}{l}1.98 \mathrm{E}-12 \sim \\
8.87 \mathrm{E}-08\end{array}$ \\
\hline & & & & & $3.28 \mathrm{Mb}$ & Hapmap40456-BTA-66218 & $48,895,237$ & FY & $1.35 E-08$ \\
\hline & & & & & $3.75 \mathrm{Mb}$ & BFGL-NGS-117323 & $49,357,135$ & MY,FY,PY,FP & $\begin{array}{l}4.32 \mathrm{E}-19 \sim \\
3.58 \mathrm{E}-08\end{array}$ \\
\hline \multirow[t]{2}{*}{ PPP1R3B } & - & & & $\begin{array}{l}\text { Chr27: 24316623-24, } \\
317,477\end{array}$ & $\begin{array}{l}259.32 \\
\mathrm{~Kb}\end{array}$ & ARS-BFGL-NGS-43776 & $24,576,801$ & $\begin{array}{l}\text { MY,FY,PY, } \\
\text { FP,PP }\end{array}$ & $\begin{array}{l}6.30 \mathrm{E}-26 \sim \\
1.20 \mathrm{E}-07\end{array}$ \\
\hline & & & & & $4.07 \mathrm{Mb}$ & BTB-01063707 & $28,386,241$ & $\begin{array}{l}\text { MY,FY,PY, } \\
F P, P P\end{array}$ & $\begin{array}{l}1.55 \mathrm{E}-45 \sim \\
1.35 \mathrm{E}-17\end{array}$ \\
\hline GALE & + & & & $\begin{array}{l}\text { Chr2:129706509-129, } \\
\text { 711,871 }\end{array}$ & $1.27 \mathrm{Mb}$ & BTA-31250-no-rs & $\begin{array}{l}128,437 \\
731\end{array}$ & $P Y, F P, P P$ & $\begin{array}{l}1.26 \mathrm{E}-24 \sim \\
3.25 \mathrm{E}-08\end{array}$ \\
\hline \multirow[t]{4}{*}{ SDS } & + & & & $\begin{array}{l}\text { Chr17: 63302876-63, } \\
311,105\end{array}$ & $\begin{array}{l}162.16 \\
\mathrm{~Kb}\end{array}$ & BFGL-NGS-110646 & $63,140,712$ & FY,PY,FP,PP & $\begin{array}{l}2.61 \mathrm{E}-14 \sim \\
3.54 \mathrm{E}-08\end{array}$ \\
\hline & & & & & $\begin{array}{l}230.59 \\
\mathrm{~Kb}\end{array}$ & Hapmap52830-rs29014800 & $63,541,690$ & $F P, P P$ & $\begin{array}{l}4.31 \mathrm{E}-11 \sim \\
3.38 \mathrm{E}-07\end{array}$ \\
\hline & & & & & $1.41 \mathrm{Mb}$ & BTB-00682411 & $61,891,068$ & PP & 2.65E-08 \\
\hline & & & & & $1.76 \mathrm{Mb}$ & BTB-01992588 & $65,071,599$ & $M Y, F Y, P Y$ & $3.55 \mathrm{E}-15 \sim$ \\
\hline
\end{tabular}


Table 3 Detailed information on the nearest and most significant SNPs from previous GWAS to the 22 differentially expressed genes in bovine liver at different lactation stages (Continued)

\begin{tabular}{|c|c|c|c|c|c|c|c|c|c|}
\hline \multirow{2}{*}{$\begin{array}{l}\text { Gene } \\
\text { name }\end{array}$} & \multirow{2}{*}{$\begin{array}{l}\text { Early } \\
\text { vs. } \\
\text { dry }\end{array}$} & \multirow{2}{*}{$\begin{array}{l}\text { Peak } \\
\text { vs. } \\
\text { dry }\end{array}$} & \multirow{2}{*}{$\begin{array}{l}\text { Peak } \\
\text { vs. } \\
\text { early }\end{array}$} & \multirow[t]{2}{*}{ Gene position $(b p)^{a}$} & \multicolumn{3}{|c|}{ Nearest and most significant SNPS of GWAS } & \multirow[t]{2}{*}{ Traits } & \multirow[t]{2}{*}{ Raw $p$ value } \\
\hline & & & & & Distance & Name & $\begin{array}{l}\text { Position } \\
(\mathrm{bp})^{\mathrm{b}}\end{array}$ & & \\
\hline & & & & & & & & & $2.01 \mathrm{E}-10$ \\
\hline & & & & & $2.93 \mathrm{Mb}$ & ARS-BFGL-NGS-17192 & $66,236,750$ & MY,PY & $\begin{array}{l}2.97 \mathrm{E}-15 \sim \\
1.30 \mathrm{E}-12\end{array}$ \\
\hline & & & & & $3.31 \mathrm{Mb}$ & ARS-BFGL-NGS-34106 & $66,619,908$ & $F Y, F P, P P$ & $\begin{array}{l}4.65 \mathrm{E}-25 \sim \\
3.68 \mathrm{E}-14\end{array}$ \\
\hline & & & & & $3.36 \mathrm{Mb}$ & Hapmap40427-BTA-41914 & $66,671,839$ & $\mathrm{FP}, \mathrm{PP}$ & $\begin{array}{l}\text { 6.15E-19 } \\
3.07 \mathrm{E}-11\end{array}$ \\
\hline \multirow[t]{2}{*}{ FBP2 } & + & + & & $\begin{array}{l}\text { Chr8:82396095-82,438, } \\
817\end{array}$ & $1.45 \mathrm{Mb}$ & BTA-14515-no-rs & $83,888,935$ & $F Y, P Y, P P$ & $\begin{array}{l}6.36 \mathrm{E}-12 \sim \\
7.78 \mathrm{E}-09\end{array}$ \\
\hline & & & & & $3.95 \mathrm{Mb}$ & ARS-BFGL-NGS-20324 & $86,384,898$ & FY,PY,FP,PP & $\begin{array}{l}7.96 \mathrm{E}-22 \sim \\
3.80 \mathrm{E}-14\end{array}$ \\
\hline$P K L R$ & - & & & $\begin{array}{l}\text { Chr3:15396974-15,408, } \\
994\end{array}$ & $1.31 \mathrm{Mb}$ & UA-IFASA-8925 & $14,084,731$ & PY & $3.48 \mathrm{E}-07$ \\
\hline SGLT1 & - & & & $\begin{array}{l}\text { Chr17: } 72690558-72, \\
741,954\end{array}$ & $3.96 \mathrm{Mb}$ & BTA-90512-no-rs & $68,732,546$ & FP,PP & $\begin{array}{l}6.81 \mathrm{E}-19 \sim \\
4.33 \mathrm{E}-13\end{array}$ \\
\hline \multirow[t]{6}{*}{ GK } & + & & & $\begin{array}{l}\text { ChrX:118110570-118, } \\
186,827\end{array}$ & $2.14 \mathrm{Mb}$ & $\begin{array}{l}\text { Hapmap34068-BES2_Contig513_ } \\
1168\end{array}$ & $\begin{array}{l}115,968 \\
607\end{array}$ & $\begin{array}{l}\text { MY,FY,PY, } \\
F P, P P\end{array}$ & $\begin{array}{l}2.30 \mathrm{E}-27 \sim \\
6.86 \mathrm{E}-09\end{array}$ \\
\hline & & & & & $2.47 \mathrm{Mb}$ & BTA-30585-no-rs & $\begin{array}{l}120,654 \\
979\end{array}$ & FY,FP,PP & $\begin{array}{l}2.02 \mathrm{E}-16 \sim \\
1.36 \mathrm{E}-08\end{array}$ \\
\hline & & & & & $2.50 \mathrm{Mb}$ & Hapmap39109-BTA-30591 & $\begin{array}{l}120,691 \\
496\end{array}$ & $\mathrm{FP}, \mathrm{PP}$ & $\begin{array}{l}6.01 \mathrm{E}-13 \sim \\
1.28 \mathrm{E}-07\end{array}$ \\
\hline & & & & & $2.89 \mathrm{Mb}$ & Hapmap33343-BTA-111447 & $\begin{array}{l}121,076 \\
442\end{array}$ & FY,PY,FP,PP & $\begin{array}{l}\text { 6.03E-13 } \\
7.03 \mathrm{E}-08\end{array}$ \\
\hline & & & & & $3.41 \mathrm{Mb}$ & BTA-98858-no-rs & $\begin{array}{l}121,600 \\
055\end{array}$ & FY,FP,PP & $\begin{array}{l}7.03 \mathrm{E}-21 \sim \\
1.01 \mathrm{E}-09\end{array}$ \\
\hline & & & & & $3.79 \mathrm{Mb}$ & Hapmap45528-BTA-102311 & $\begin{array}{l}114,319 \\
576\end{array}$ & $\mathrm{FY}, \mathrm{FP}, \mathrm{PP}$ & $\begin{array}{l}3.01 \mathrm{E}-18 \sim \\
5.13 \mathrm{E}-09\end{array}$ \\
\hline \multirow[t]{2}{*}{ CYPTA1 } & + & + & & $\begin{array}{l}\text { Chr14:26348324-26, } \\
358,692\end{array}$ & $3.76 \mathrm{Mb}$ & ARS-BFGL-BAC-12159 & $22,587,081$ & MY,FY,PY,PP & $\begin{array}{l}4.94 \mathrm{E}-24 \sim \\
6.01 \mathrm{E}-11\end{array}$ \\
\hline & & & & & $4.41 \mathrm{Mb}$ & ARS-BFGL-NGS-3198 & $21,933,950$ & FY,PY,FP,PP & $\begin{array}{l}1.21 \mathrm{E}-25 \sim \\
1.45 \mathrm{E}-12\end{array}$ \\
\hline \multirow[t]{4}{*}{ ANGPTL4 } & + & + & & $\begin{array}{l}\text { Chr7:18236482-18,243, } \\
588\end{array}$ & $1.84 \mathrm{Mb}$ & Hapmap23865-BTA-111820 & $16,397,706$ & PP & $1.51 \mathrm{E}-08$ \\
\hline & & & & & $4.60 \mathrm{Mb}$ & BFGL-NGS-119066 & $13,632,174$ & $\mathrm{FP}, \mathrm{PP}$ & $\begin{array}{l}9.49 \mathrm{E}-20 \sim \\
7.48 \mathrm{E}-10\end{array}$ \\
\hline & & & & & $4.63 \mathrm{Mb}$ & ARS-BFGL-NGS-109534 & $13,608,935$ & $\mathrm{FP}, \mathrm{PP}$ & $\begin{array}{l}8.42 \mathrm{E}-20 \sim \\
1.94 \mathrm{E}-09\end{array}$ \\
\hline & & & & & $4.65 \mathrm{Mb}$ & BFGL-NGS-111315 & $13,584,721$ & $\mathrm{FY}, \mathrm{PY}, \mathrm{FP}, \mathrm{PP}$ & $\begin{array}{l}4.90 \mathrm{E}-29 \sim \\
7.03 \mathrm{E}-12\end{array}$ \\
\hline \multirow[t]{2}{*}{ IGF-1R } & - & - & & $\begin{array}{l}\text { Chr21:7967718-8,268, } \\
246\end{array}$ & $\begin{array}{l}769.19 \\
\mathrm{~Kb}\end{array}$ & ARS-BFGL-NGS-10704 & $9,037,431$ & $\mathrm{FP}, \mathrm{PP}$ & $\begin{array}{l}2.60 \mathrm{E}-13 \sim \\
4.77 \mathrm{E}-08\end{array}$ \\
\hline & & & & & $1.07 \mathrm{Mb}$ & ARS-BFGL-NGS-77061 & $6,900,116$ & PP & $9.32 \mathrm{E}-10$ \\
\hline \multirow[t]{3}{*}{ CDKN1A } & - & & + & $\begin{array}{l}\text { Chr23:10551755-10, } \\
\text { 568,782 }\end{array}$ & $2.68 \mathrm{Mb}$ & BFGL-NGS-115177 & $7,874,236$ & MY,FY,PY & $\begin{array}{l}1.83 \mathrm{E}-24 \sim \\
6.49 \mathrm{E}-17\end{array}$ \\
\hline & & & & & $3.28 \mathrm{Mb}$ & ARS-BFGL-NGS-41214 & $13,846,320$ & MY,FY,PY,PP & $\begin{array}{l}2.10 \mathrm{E}-24 \sim \\
3.13 \mathrm{E}-11\end{array}$ \\
\hline & & & & & $4.20 \mathrm{Mb}$ & ARS-BFGL-NGS-17887 & $14,769,068$ & MY,FY,PY,PP & $\begin{array}{l}3.76 \mathrm{E}-24 \sim \\
6.86 \mathrm{E}-09\end{array}$ \\
\hline ODC1 & + & + & & $\begin{array}{l}\text { Chr11:87175389-87, } \\
182,661\end{array}$ & $74.93 \mathrm{~Kb}$ & BTA-1 10370-no-rs & $87,257,595$ & $F Y, P Y, F P$ & $\begin{array}{l}3.10 \mathrm{E}-12 \sim \\
2.68 \mathrm{E}-07\end{array}$ \\
\hline
\end{tabular}


Table 3 Detailed information on the nearest and most significant SNPs from previous GWAS to the 22 differentially expressed genes in bovine liver at different lactation stages (Continued)

\begin{tabular}{|c|c|c|c|c|c|c|c|c|c|}
\hline \multirow{2}{*}{$\begin{array}{l}\text { Gene } \\
\text { name }\end{array}$} & \multirow{2}{*}{$\begin{array}{l}\text { Early } \\
\text { vs. } \\
\text { dry }\end{array}$} & \multirow{2}{*}{$\begin{array}{l}\text { Peak } \\
\text { vs. } \\
\text { dry }\end{array}$} & \multirow{2}{*}{$\begin{array}{l}\text { Peak } \\
\text { vs. } \\
\text { early }\end{array}$} & \multirow[t]{2}{*}{ Gene position (bp) $)^{a}$} & \multicolumn{3}{|c|}{ Nearest and most significant SNPs of GWAS } & \multirow[t]{2}{*}{ Traits } & \multirow[t]{2}{*}{ Raw $p$ value } \\
\hline & & & & & Distance & Name & $\begin{array}{l}\text { Position } \\
(\mathrm{bp})^{\mathrm{b}}\end{array}$ & & \\
\hline & & & & & $96.35 \mathrm{~Kb}$ & BFGL-NGS-114578 & $87,279,008$ & FY,PY & $\begin{array}{l}2.85 \mathrm{E}-11 \sim \\
1.82 \mathrm{E}-08\end{array}$ \\
\hline & & & & & $\begin{array}{l}197.94 \\
\mathrm{~Kb}\end{array}$ & ARS-BFGL-NGS-42014 & $87,380,604$ & FY & 9.34E-09 \\
\hline & & & & & $\begin{array}{l}249.36 \\
\mathrm{~Kb}\end{array}$ & ARS-BFGL-NGS-17731 & $86,926,025$ & FY,PY,FP & $\begin{array}{l}3.31 \mathrm{E}-16 \sim \\
3.55 \mathrm{E}-07\end{array}$ \\
\hline & & & & & $\begin{array}{l}605.73 \\
\mathrm{~Kb}\end{array}$ & Hapmap53648-rs29021240 & $86,569,656$ & FY,PY & $\begin{array}{l}1.00 \mathrm{E}-10 \sim \\
2.14 \mathrm{E}-08\end{array}$ \\
\hline & & & & & $\begin{array}{l}641.51 \\
\mathrm{~Kb}\end{array}$ & ARS-BFGL-NGS-70263 & $87,824,167$ & MY,FY,PY,PP & $\begin{array}{l}1.16 \mathrm{E}-17 \sim \\
2.25 \mathrm{E}-08\end{array}$ \\
\hline & & & & & $\begin{array}{l}789.42 \\
\mathrm{~Kb}\end{array}$ & ARS-BFGL-NGS-41670 & $87,972,079$ & FY,PP & $\begin{array}{l}1.41 \mathrm{E}-08 \sim \\
2.53 \mathrm{E}-07\end{array}$ \\
\hline & & & & & $\begin{array}{l}817.29 \\
\mathrm{~Kb}\end{array}$ & ARS-BFGL-BAC-16207 & $87,999,946$ & FY,PY,FP,PP & $\begin{array}{l}4.08 \mathrm{E}-10 \sim \\
3.65 \mathrm{E}-07\end{array}$ \\
\hline & & & & & $2.02 \mathrm{Mb}$ & Hapmap52066-rs29015690 & $85,153,576$ & FY & $1.10 \mathrm{E}-07$ \\
\hline & & & & & $2.16 \mathrm{Mb}$ & ARS-BFGL-NGS-28030 & $89,338,824$ & FY,PY & $\begin{array}{l}2.08 \mathrm{E}-12 \sim \\
1.33 \mathrm{E}-07\end{array}$ \\
\hline & & & & & $2.19 \mathrm{Mb}$ & Hapmap31724-BTA-126967 & $89,371,911$ & $F Y, P Y, F P$ & $\begin{array}{l}1.05 \mathrm{E}-15 \sim \\
1.43 \mathrm{E}-08\end{array}$ \\
\hline & & & & & $2.30 \mathrm{Mb}$ & ARS-BFGL-NGS-2015 & $84,872,349$ & FY,PY,FP,PP & $\begin{array}{l}1.83 \mathrm{E}-18 \sim \\
6.84 \mathrm{E}-05\end{array}$ \\
\hline & & & & & $2.35 \mathrm{Mb}$ & ARS-BFGL-NGS-104652 & $84,828,071$ & MY,FY,PY & $\begin{array}{l}1.05 \mathrm{E}-18 \sim \\
2.22 \mathrm{E}-09\end{array}$ \\
\hline & & & & & $3.54 \mathrm{Mb}$ & ARS-BFGL-NGS-63978 & $83,637,998$ & FY,PY,FP,PP & $\begin{array}{l}2.27 \mathrm{E}-15 \sim \\
1.78 \mathrm{E}-07\end{array}$ \\
\hline & & & & & $3.65 \mathrm{Mb}$ & Hapmap40477-BTA-105881 & $83,526,455$ & FY,PY,FP,PP & $\begin{array}{l}1.80 \mathrm{E}-24 \sim \\
2.41 \mathrm{E}-11\end{array}$ \\
\hline & & & & & $4.19 \mathrm{Mb}$ & Hapmap56387-rs29014077 & $82,982,779$ & FY & $1.25 \mathrm{E}-07$ \\
\hline & & & & & $4.25 \mathrm{Mb}$ & ARS-BFGL-NGS-93601 & $82,920,732$ & $F Y, P Y, F P, P P$ & $\begin{array}{l}1.88 \mathrm{E}-21 \sim \\
2.96 \mathrm{E}-13\end{array}$ \\
\hline & & & & & $4.53 \mathrm{Mb}$ & Hapmap46768-BTA-117394 & $82,643,114$ & MY,FY,PY & $\begin{array}{l}1.78 \mathrm{E}-13 \sim \\
1.73 \mathrm{E}-07\end{array}$ \\
\hline GADD45B & & + & + & $\begin{array}{l}\text { Chr7:22411968-22,414, } \\
079\end{array}$ & $2.24 \mathrm{Mb}$ & Hapmap49309-BTA-78604 & $24,655,689$ & PP & 4.34E-14 \\
\hline \multirow[t]{8}{*}{ LPIN1 } & & + & + & $\begin{array}{l}\text { Chr11:86050742-86, } \\
128,538\end{array}$ & $2.38 \mathrm{~Kb}$ & ARS-BFGL-NGS-14236 & $86,048,363$ & FY,PY & $\begin{array}{l}1.29 \mathrm{E}-12 \sim \\
4.60 \mathrm{E}-08\end{array}$ \\
\hline & & & & & $\begin{array}{l}441.12 \\
\mathrm{~Kb}\end{array}$ & Hapmap53648-rs29021240 & $86,569,656$ & FY,PY & $\begin{array}{l}1.00 \mathrm{E}-10 \sim \\
2.14 \mathrm{E}-08\end{array}$ \\
\hline & & & & & $\begin{array}{l}797.49 \\
\mathrm{~Kb}\end{array}$ & ARS-BFGL-NGS-17731 & $86,926,025$ & FY,PY,FP & $\begin{array}{l}3.31 \mathrm{E}-16 \sim \\
3.55 \mathrm{E}-07\end{array}$ \\
\hline & & & & & $\begin{array}{l}897.17 \\
\mathrm{~Kb}\end{array}$ & Hapmap52066-rs29015690 & $85,153,576$ & FY & 1.10E-07 \\
\hline & & & & & $1.13 \mathrm{Mb}$ & BTA-1 10370-no-rs & $87,257,595$ & FY,PY,FP & $\begin{array}{l}3.10 \mathrm{E}-12 \sim \\
2.68 \mathrm{E}-07\end{array}$ \\
\hline & & & & & $1.15 \mathrm{Mb}$ & BFGL-NGS-114578 & $87,279,008$ & FY,PY & $\begin{array}{l}2.85 \mathrm{E}-11 \sim \\
1.82 \mathrm{E}-08\end{array}$ \\
\hline & & & & & $1.18 \mathrm{Mb}$ & ARS-BFGL-NGS-2015 & $84,872,349$ & FY,PY,FP,PP & $\begin{array}{l}1.83 \mathrm{E}-18 \sim \\
6.84 \mathrm{E}-05\end{array}$ \\
\hline & & & & & $1.22 \mathrm{Mb}$ & ARS-BFGL-NGS-104652 & $84,828,071$ & $M Y, F Y, P Y$ & $\begin{array}{l}1.05 \mathrm{E}-18 \sim \\
2.22 \mathrm{E}-09\end{array}$ \\
\hline
\end{tabular}


Table 3 Detailed information on the nearest and most significant SNPs from previous GWAS to the 22 differentially expressed genes in bovine liver at different lactation stages (Continued)

\begin{tabular}{|c|c|c|c|c|c|c|c|c|c|}
\hline \multirow{2}{*}{$\begin{array}{l}\text { Gene } \\
\text { name }\end{array}$} & \multirow{2}{*}{$\begin{array}{l}\text { Early } \\
\text { vs. } \\
\text { dry }\end{array}$} & \multirow{2}{*}{$\begin{array}{l}\text { Peak } \\
\text { vs. } \\
\text { dry }\end{array}$} & \multirow{2}{*}{$\begin{array}{l}\text { Peak } \\
\text { vs. } \\
\text { early }\end{array}$} & \multirow[t]{2}{*}{ Gene position (bp) ${ }^{a}$} & \multicolumn{3}{|c|}{ Nearest and most significant SNPs of GWAS } & \multirow[t]{2}{*}{ Traits } & \multirow[t]{2}{*}{ Raw $p$ value } \\
\hline & & & & & Distance & Name & $\begin{array}{l}\text { Position } \\
(\mathrm{bp})^{\mathrm{b}}\end{array}$ & & \\
\hline & & & & & $1.25 \mathrm{Mb}$ & ARS-BFGL-NGS-42014 & $87,380,604$ & FY & 9.34E-09 \\
\hline & & & & & $1.70 \mathrm{Mb}$ & ARS-BFGL-NGS-70263 & $87,824,167$ & MY,FY,PY,PP & $\begin{array}{l}1.16 \mathrm{E}-17 \sim \\
2.25 \mathrm{E}-08\end{array}$ \\
\hline & & & & & $1.84 \mathrm{Mb}$ & ARS-BFGL-NGS-41670 & $87,972,079$ & FY,PP & $\begin{array}{l}1.41 \mathrm{E}-08 \sim \\
2.53 \mathrm{E}-07\end{array}$ \\
\hline & & & & & $1.87 \mathrm{Mb}$ & ARS-BFGL-BAC-16207 & $87,999,946$ & FY,PY,FP,PP & $\begin{array}{l}4.08 \mathrm{E}-10 \sim \\
3.65 \mathrm{E}-07\end{array}$ \\
\hline & & & & & $2.41 \mathrm{Mb}$ & ARS-BFGL-NGS-63978 & $83,637,998$ & FY,PY,FP,PP & $\begin{array}{l}2.27 \mathrm{E}-15 \sim \\
1.78 \mathrm{E}-07\end{array}$ \\
\hline & & & & & $2.52 \mathrm{Mb}$ & Hapmap40477-BTA-105881 & $83,526,455$ & FY,PY,FP,PP & $\begin{array}{l}1.80 \mathrm{E}-24 \sim \\
2.41 \mathrm{E}-11\end{array}$ \\
\hline & & & & & $3.07 \mathrm{Mb}$ & Hapmap56387-rs29014077 & $82,982,779$ & $\mathrm{FY}$ & $1.25 \mathrm{E}-07$ \\
\hline & & & & & $3.13 \mathrm{Mb}$ & ARS-BFGL-NGS-93601 & $82,920,732$ & FY,PY,FP,PP & $\begin{array}{l}1.88 \mathrm{E}-21 \sim \\
2.96 \mathrm{E}-13\end{array}$ \\
\hline & & & & & $3.21 \mathrm{Mb}$ & ARS-BFGL-NGS-28030 & $89,338,824$ & FY,PY & $\begin{array}{l}2.08 \mathrm{E}-12 \sim \\
1.33 \mathrm{E}-07\end{array}$ \\
\hline & & & & & $3.24 \mathrm{Mb}$ & Hapmap31724-BTA-126967 & $89,371,911$ & FY,PY,FP & $\begin{array}{l}1.05 \mathrm{E}-15 \sim \\
1.43 \mathrm{E}-08\end{array}$ \\
\hline & & & & & $3.41 \mathrm{Mb}$ & Hapmap46768-BTA-117394 & $82,643,114$ & MY,FY,PY & $\begin{array}{l}1.78 \mathrm{E}-13 \sim \\
1.73 \mathrm{E}-07\end{array}$ \\
\hline & & & & & $4.21 \mathrm{Mb}$ & ARS-BFGL-NGS-105586 & $81,845,043$ & $F Y, P Y, F P$ & $\begin{array}{l}2.47 \mathrm{E}-15 \sim \\
3.00 \mathrm{E}-08\end{array}$ \\
\hline & & & & & $4.23 \mathrm{Mb}$ & ARS-BFGL-NGS-52709 & $81,819,453$ & FY,PY,FP,PP & $\begin{array}{l}3.01 \mathrm{E}-25 \sim \\
1.28 \mathrm{E}-08\end{array}$ \\
\hline \multirow[t]{5}{*}{ DUSP1 } & & + & & $\begin{array}{l}\text { Chr20:4449106-4,452, } \\
189\end{array}$ & $44.19 \mathrm{~Kb}$ & ARS-BFGL-NGS-48030 & $4,496,376$ & PP & $2.07 \mathrm{E}-08$ \\
\hline & & & & & $53.45 \mathrm{~Kb}$ & Hapmap54098-rs29010434 & $4,395,656$ & FY,PY,PP & $\begin{array}{l}1.35 \mathrm{E}-12 \sim \\
1.66 \mathrm{E}-08\end{array}$ \\
\hline & & & & & $3.32 \mathrm{Mb}$ & Hapmap49207-BTA-51446 & $7,770,495$ & $\begin{array}{l}\text { MY,FY,PY, } \\
\text { FP,PP }\end{array}$ & $\begin{array}{l}5.02 \mathrm{E}-27 \sim \\
9.06 \mathrm{E}-11\end{array}$ \\
\hline & & & & & $3.50 \mathrm{Mb}$ & $\begin{array}{l}\text { Hapmap36217-SCAF } \\
\text { FOLD290026_21689 }\end{array}$ & $7,954,374$ & FY,PY & $\begin{array}{l}7.19 \mathrm{E}-09 \sim \\
7.36 \mathrm{E}-09\end{array}$ \\
\hline & & & & & $3.74 \mathrm{Mb}$ & ARS-BFGL-NGS-27058 & 712,232 & FY,PY,FP & $\begin{array}{l}1.11 \mathrm{E}-10 \sim \\
3.92 \mathrm{E}-07\end{array}$ \\
\hline LMNA & + & & & $\begin{array}{l}\text { Chr3:14695234-14,724, } \\
663\end{array}$ & $0.61 \mathrm{Mb}$ & UA-IFASA-8925 & $14,084,731$ & PY & $3.48 \mathrm{E}-07$ \\
\hline
\end{tabular}

a,b Means the position on the bovine genome sequence of UMD3.1.80

$M Y$ milk yield; $F Y$ milk fat yield; $P Y$ milk protein yield; $F P$ milk fat percentage; $P P$ milk protein percentage.

before calving) mainly participated in metabolisms of lipid, fatty acid, protein, carbohydrate and energy, and involved in MAPK, p53 and PPAR signaling pathways. Especially, lipid metabolism was significantly enriched such as fat digestion and absorption, fatty acid metabolism, bile secretion, endocytosis, biosynthesis of unsaturated fatty acids, endocytosis, fatty acid metabolism, AMPK, and PPAR signaling pathways. This is mostly likely due to milk lipids, proteins, lactose, saturated and unsaturated fatty acids that need to be synthesized during lactation. These results also suggested that metabolism and synthesis in liver provided nutrient substance support for the milking in mammary gland probably through the milking vein in dairy cows. Previous studies also revealed that the contents of triacylglycerol [26] and blood nonesterified fatty acids (NEFA) [27] were increased greatly during the perinatal period.

Based on the integrated analysis of DEGs, QTLs, GWAS data and biological functions, a total of ten promising candidate genes were found. Among them, four genes like GALE, ANGPTL4, LPIN1, and CDKN1A have been reported to play roles in milk production [28]. 
Six novel candidate genes identified in this study included APOC2, PPP1R3B, PKLR, ODC1, DUSP1, and LMNA.

APOC2, encoded by the $A P O C 2$ gene, is the component of chylomicrons $(\mathrm{CM})$, very low density lipoprotein (VLDLs), low density lipoprotein (LDLs) and high density lipoprotein (HDLs) in plasma. It plays an important role in lipoprotein metabolism as an activator of lipoprotein lipase. The deficiency of $A P O C 2$ could cause high circulating levels of triglycerides (TGs), while overexpression of $A P O C 2$ could inhibit lipoprotein lipase activity $[29,30]$. In addition, $A P O C 2$ was close to two known QTLs for milk protein yield and milk yield with distances of 1.9 to $2.3 \mathrm{cM}$ and also near four significant SNPs for milk fat and protein traits detected by Cole et al.(2011) [23].

$P P P 1 R 3 B$ and $P K L R$ are related to carbohydrate metabolism. PPP1R3B activates glycogen synthesis and limits glycogen breakdown in the liver and skeletal muscle [31]. It was not only close to one reported QTL for milk fat percentage with distance of $0.5 \mathrm{cM}$, also near two significant SNPs for milk fat and protein identified by GWAS [23]. PKLR plays a key role in hepatic glycolysis [32]. It was close to three known QTLs (0.7 to 1.7 $\mathrm{cM}$ ) for milk fat yield, milk protein percentage and milk fat percentage and also near one significant SNP for milk protein yield [23].

The ODC1 gene encodes ODC1 that is a key enzyme of polyamine biosynthesis and a main regulator of protein synthesis and lactogenesis. The transcription of $O D C 1$ is stimulated by high nutritional levels and is elevated during periods of rapid mammary growth and differentiation [33]. ODC1 was very close to two known QTLs for milk fat yield with $0.1 \mathrm{cM}$ and near 18 significant SNPs for milk fat and protein with distances $74 \mathrm{~Kb} \sim 4.53 \mathrm{Mb}$ [23].

DUSP1 is involved in the epithelial-to-mesenchymal transition, regulation of breast cancer stem cells (CSCs) and signal transduction [34]. DUSP1 was not only close to the two known QTLs for milk fat yield and milk protein yield $(2.7$ to $4.6 \mathrm{cM})$ but also near five significant SNPs for milk fat and protein [23]. LMNA plays an important role in nuclear assembly, chromatin organization, nuclear membrane formation and telomere dynamics. The mutations in $L M N A$ caused an imbalance between lipid oxidation and oxidative glucose metabolism in skeletal muscle metabolism [35]. It was near to the peak positions of three QTLs $(0.5$ to $2.9 \mathrm{cM})$ for milk fat yield, milk protein percentage and milk fat percentage and near one significant SNP for milk protein yield [23].

\section{Conclusions}

This study detected significantly expressed genes (DEGs) in liver among three lactation periods (dry period, early and peak of lactation) by performing RNA sequencing in
Holstein cows. Integrated analysis of DEGs, previously reported QTL and GWAS data, and biological functions of genes identified 10 promising candidate genes for milk production traits, including $A P O C 2, P P P 1 R 3 B$, PKLR, ODC1, DUSP1, LMNA, GALE, ANGPTL4, LPIN1 and CDKN1A. Our findings provided a solid basis for further in-depth studies on how these genes regulate milk synthesis and molecular information for genomic selection in dairy cattle.

\section{Supplementary information}

Supplementary information accompanies this paper at https://doi.org/10. 1186/s12863-020-00882-y.

Additional file 1. The basic statistics for RNA-seq reads generated from liver tissues of three cows at different lactation stages.

Additional file 2. Summary of sequence read alignments to the reference genome.

Additional file 3. Correlation analysis of the reads.

Additional file 4. Volcano plot displaying differential expressed genes in bovine liver at different lactation stages.

Additional file 5. List of differentially expressed genes in bovine liver between early lactation and dry period/peak of lactation and dry period/ peak of lactation and early lactation.

Additional file 6. List of significant GO terms assignment for DEGs in bovine liver between early lactation and dry period/peak of lactation and dry period/peak of lactation and early lactation.

Additional file 7. List of significant KEGG pathway categories for DEGs in bovine liver between early lactation and dry period/peak of lactation and dry period/peak of lactation and early lactation.

\section{Abbreviations}

QTL: Quantitative trait loci; GWAS: Genome-wide association study; RNAseq: RNA sequencing; LA: Linkage analysis; LD: Linkage disequilibrium; DEGs: Differentially expressed genes; EGs: Expressed genes; NEFA: Nonesterified fatty acids; CM: Chylomicrons; VLDL: Very low density lipoprotein; LDL: Low density lipoprotein; HDL: High density lipoprotein

\section{Acknowledgements}

We appreciate Baoding Hongda Animal Husbandry Limited Company for providing Chinese Holstein cows.

\section{Authors' contributions}

$J \mathrm{~L}$ and DS conceived and designed this experiment, $Y L, Y G$ and $Q L$ collected the liver samples from cows, QL, RL and $Y Y$ isolated the RNA samples, QL performed the RNA-related experiments, data analysis, and the manuscript was prepared by $\mathrm{QL}, \mathrm{RL}, \mathrm{DS}$, and $\mathrm{J}$. All authors read and approved the final manuscript.

\section{Funding}

This work was financially supported by China Modern Agro-industry Technology Research System (CARS-36), Research Program of Hebei Province (16226604D), Hebei Dairy Cattle Innovation Team of Modern Agro-industry Technology Research System (HBCT2018120203), National Natural Science Foundation of China (31872330), and the Program for Changjiang Scholar and Innovation Research Team in University (IRT_15R62). The funders had no role in study design, data collection, and analysis, or in the decision to publish or in preparing the paper.

Availability of data and materials

All relevant data are available within the article and its additional files. The RNA sequencing datasets generated during current study are not publicly available because another program is under way, but are available from the corresponding author on reasonable request. 


\section{Ethics approval and consent to participate}

All protocols for collection of the tissues of experimental individuals and phenotypic observations were reviewed and approved by the Animal Welfare Committee of Hebei Agricultural University. Tissue samples were collected specifically for this study following standard procedures with the written consent from the Baoding Hongda Animal Husbandry Limited Company who owned the Chinese Holstein cows.

\section{Consent for publication}

Not applicable.

\section{Competing interests}

Dongxiao Sun is a member of the editorial board (Associate Editor) of this journal. Dongxiao Sun and all the other authors declare that they have no competing interests.

\section{Author details}

${ }^{1}$ College of Animal Science and Technology, Hebei Agricultural University, Lekai South Street, Baoding 071001, China. ${ }^{2}$ Hebei Animal Husbandry and Veterinary Institute, Baoding 071000, China. ${ }^{3}$ Department of Animal Genetics, Breeding and Reproduction, College of Animal Science and Technology, Key Laboratory of Animal Genetics and Breeding of Ministry of Agriculture, National Engineering Laboratory of Animal Breeding, China Agricultural University, No.2 Yuanmingyuan West Road, Beijing 100193, China. ${ }^{4}$ College of Veterinary Medicine, Hebei Agricultural University, Baoding 071001, China.

Received: 29 December 2019 Accepted: 1 July 2020

Published online: 09 July 2020

\section{References}

1. Georges M, Nielsen D, Mackinnon M, Mishra A, Okimoto R, Pasquino AT Sargeant LS, Sorensen A, Steele MR, Zhao X, Et A. Mapping quantitative trait loci controlling milk production in dairy cattle by exploiting progeny testing. Genetics. 1995;139(2):907-20.

2. Grisart B, Farnir F, Karim L, Cambisano N, Kim JJ, Kvasz A, Mni M, Simon P, Frere JM, Coppieters W, Georges M. Genetic and functional confirmation of the causality of the DGAT1 K232A quantitative trait nucleotide in affecting milk yield and composition. Proc Natl Acad Sci U S A. 2004;101(8):2398-403.

3. Andersson L. Genome-wide association analysis in domestic animals: a powerful approach for genetic dissection of trait loci. Genetica. 2009;136(2): 341-9.

4. Schennink A, Bovenhuis $H$, Leon-Kloosterziel KM, van Arendonk JA, Visker $\mathrm{MH}$. Effect of polymorphisms in the FASN, OLR1, PPARGC1A, PRL and STAT5A genes on bovine milk-fat composition. Anim Genet. 2009;40(6):90916.

5. Blott S, Kim JJ, Moisio S, Schmidt-Kuntzel A, Cornet A, Berzi P, Cambisano N, Ford C, Grisart B, Johnson D, Karim L, Simon P, Snell R, Spelman R, Wong J, Vilkki J, Georges M, Farnir F, Coppieters W. Molecular dissection of a quantitative trait locus: a phenylalanine-to-tyrosine substitution in the transmembrane domain of the bovine growth hormone receptor is associated with a major effect on milk yield and composition. Genetics. 2003;163(1):253-66.

6. Cohen-Zinder M, Seroussi E, Larkin DM, Loor JJ, Everts-Van DWA, Lee JH, Drackley JK, Band MR, Hernandez AG, Shani M, Lewin HA, Weller Jl, Ron M. Identification of a missense mutation in the bovine ABCG2 gene with a major effect on the QTL on chromosome 6 affecting milk yield and composition in Holstein cattle. Genome Res. 2005;15(7):936-44.

7. Canovas A, Rincon G, Islas-Trejo A, Wickramasinghe S, Medrano JF. SNP discovery in the bovine milk transcriptome using RNA-Seq technology. Mamm Genome. 2010;21(11-12):592-8.

8. Cui X, Hou Y, Yang S, Xie Y, Zhang S, Zhang Y, Zhang Q, Lu X, Liu GE, Sun D. Transcriptional profiling of mammary gland in Holstein cows with extremely different milk protein and fat percentage using RNA sequencing. BMC Genomics. 2014;15:226.

9. Huang W, Khatib H. Comparison of transcriptomic landscapes of bovine embryos using RNA-Seq. BMC Genomics. 2010;11:711.

10. Huang W, Nadeem A, Zhang B, Babar M, Soller M, Khatib H. Characterization and comparison of the leukocyte transcriptomes of three cattle breeds. PLoS One. 2012;7(1):e30244.
11. Scholey RA, Evans NJ, Blowey RW, Massey JP, Murray RD, Smith RF, Ollier WE, Carter SD. Identifying host pathogenic pathways in bovine digital dermatitis by RNA-Seq analysis. Vet J. 2013;197(3):699-706.

12. Mccabe M, Waters S, Morris D, Kenny D, Lynn D, Creevey C. RNA-seq analysis of differential gene expression in liver from lactating dairy cows divergent in negative energy balance. BMC Genomics. 2012;13:193.

13. Li C, Cai W, Zhou C, Yin H, Zhang Z, Loor JJ, Sun D, Zhang Q, Liu J, Zhang S. RNA-Seq reveals 10 novel promising candidate genes affecting milk protein concentration in the Chinese Holstein population. Sci Rep. 2016;6: 26813.

14. Mozduri Z, Bakhtiarizadeh MR, Salehi A. Integrated regulatory network reveals novel candidate regulators in the development of negative energy balance in cattle. Animal. 2018;12(6):1196-207.

15. Graber M, Kohler S, Kaufmann T, Doherr MG, Bruckmaier RM, van Dorland HA. A field study on characteristics and diversity of gene expression in the liver of dairy cows during the transition period. J Dairy Sci. 2010;93(11): 5200-15.

16. Schlegel G, Ringseis R, Keller J, Schwarz FJ, Eder K. Changes in the expression of hepatic genes involved in cholesterol homeostasis in dairy cows in the transition period and at different stages of lactation. J Dairy Sci. 2012:95(7):3826-36.

17. van Dorland HA, Richter S, Morel I, Doherr MG, Castro N, Bruckmaier RM. Variation in hepatic regulation of metabolism during the dry period and in early lactation in dairy cows. J Dairy Sci. 2009;92(5):1924-40.

18. Aschenbach JR, Kristensen NB, Donkin SS, Hammon HM, Penner GB. Gluconeogenesis in dairy cows: the secret of making sweet milk from sour dough. IUBMB Life. 2010;62(12):869-77.

19. Smith JL, Lear SR, Forte TM, Ko W, Massimi M, Erickson SK. Effect of pregnancy and lactation on lipoprotein and cholesterol metabolism in the rat. J Lipid Res. 1998:39(11):2237-49.

20. Weber C, Hametner C, Tuchscherer A, Losand B, Kanitz E, Otten W, Sauerwein H, Bruckmaier RM, Becker F, Kanitz W, Hammon HM. Hepatic gene expression involved in glucose and lipid metabolism in transition cows: effects of fat mobilization during early lactation in relation to milk performance and metabolic changes. J Dairy Sci. 2013;96(9):5670-81.

21. Bu D, Bionaz M, Wang M, Nan X, Ma L, Wang J. Transcriptome difference and potential crosstalk between liver and mammary tissue in mid-lactation primiparous dairy cows. PLoS One. 2017;12(3):e173082.

22. Liang R, Han B, Li Q, Yuan Y, Li J, Sun D. Using RNA sequencing to identify putative competing endogenous RNAs (ceRNAs) potentially regulating fat metabolism in bovine liver. Sci Rep. 2017;7(1):6396

23. Cole JB, Wiggans GR, Ma L, Sonstegard TS, Lawlor TJ, Crooker BA, Van Tassell CP, Yang J, Wang S, Matukumalli LK, Da Y. Genome-wide association analysis of thirty one production, health, reproduction and body conformation traits in contemporary U.S. Holstein cows. BMC Genomics. 2011;12:408

24. Rapaport F, Khanin R, Liang Y, Pirun M, Krek A, Zumbo P, Mason CE, Socci ND, Betel D. Comprehensive evaluation of differential gene expression analysis methods for RNA-seq data. Genome Biol. 2013;14(9):R95.

25. Trapnell C, Hendrickson DG, Sauvaqeau M, Goff L, Rinn JL, Pachter L. Differential analysis of gene regulation at transcript resolution with RNA-seq [J]. Nature Biotechnol. 2013;31(1):46.

26. Selim S, Salin S, Taponen J, Vanhatalo A, Kokkonen T, Elo KT. Prepartal dietary energy alters transcriptional adaptations of the liver and subcutaneous adipose tissue of dairy cows during the transition period. Physiol Genomics. 2014;46(9):328-37.

27. Pullen DL, Palmquist DL, Emery RS. Effect on days of lactation and methionine hydroxy analog on incorporation of plasma fatty acids into plasma triglycerides. J Dairy Sci. 1989;72(1):49-58.

28. Bionaz M, Loor JJ. Gene networks driving bovine milk fat synthesis during the lactation cycle. BMC Genomics. 2008;9:366.

29. Jong MC, Hofker MH, Havekes LM. Role of ApoCs in lipoprotein metabolism: functional differences between ApoC1, ApoC2, and ApoC3. Arterioscler Thromb Vasc Biol. 1999:19(3):472-84

30. Havel RJ, Fielding CJ, Olivecrona T, Shore VG, Fielding PE, Egelrud T. Cofactor activity of protein components of human very low density lipoproteins in the hydrolysis of triglycerides by lipoproteins lipase from different sources. Biochemistry-US. 1973;12(9):1828-33.

31. Dunn JS, Mlynarski WM, Pezzolesi MG, Borowiec M, Powers C, Krolewski AS, Doria A. Examination of PPP1R3B as a candidate gene for the type 2 
diabetes and MODY loci on chromosome 8p23. Ann Hum Genet. 2006;70(Pt 5):587-93.

32. Grala TM, Roche JR, Kay JK, Rius AG, White HM, Donkin SS, Littlejohn MD,

Snell RG, Phyn CV. The expression of genes involved in hepatic metabolism is altered by temporary changes to milking frequency. J Dairy Sci. 2014; 97(2):838-50.

33. Kim HH, Park CS. A compensatory nutrition regimen during gestation stimulates mammary development and lactation potential in rats. J Nutr. 2004;134(4):756-61.

34. Boulding T, Wu F, Mccuaig R, Dunn J, Sutton CR, Hardy K, Tu W, Bullman A, Yip D, Dahlstrom JE, Rao S. Differential roles for DUSP family members in epithelial-to-Mesenchymal transition and Cancer stem cell regulation in breast Cancer. PLoS One. 2016;11(2):e148065.

35. Boschmann M, Engeli S, Moro C, Luedtke A, Adams F, Gorzelniak K, Rahn G, Mahler A, Dobberstein K, Kruger A, Schmidt S, Spuler S, Luft FC, Smith SR, Schmidt HH, Jordan J. LMNA mutations, skeletal muscle lipid metabolism, and insulin resistance. J Clin Endocrinol Metab. 2010;95(4):1634-43.

\section{Publisher's Note}

Springer Nature remains neutral with regard to jurisdictional claims in published maps and institutional affiliations.

Ready to submit your research? Choose BMC and benefit from:

- fast, convenient online submission

- thorough peer review by experienced researchers in your field

- rapid publication on acceptance

- support for research data, including large and complex data types

- gold Open Access which fosters wider collaboration and increased citations

- maximum visibility for your research: over $100 \mathrm{M}$ website views per year

At BMC, research is always in progress.

Learn more biomedcentral.com/submissions 